\title{
Computation of Hyperbolic Geometric Flow on Closed Riemann Surfaces
}

\author{
Zheng Xie* and Zheng Ye
}

\begin{abstract}
Hyperbolic geometric flow, introduced by Kong and Liu, is a system of nonlinear evolution partial differential equations of second order for Riemannian metric and Ricci curvature. This work introduces two numerical algorithms for initial value problem of this flow on closed Riemann surfaces with circle packing metric and pointwise conformal metric, and demonstrates the evolution of metric by data and graphs. Specially, the numerical experiment and the analysis of schemes show that the Euler character is an essential obstruction to the existence of periodic solution for any initial metrics on sphere and double torus.
\end{abstract}

Keywords: Hyperbolic geometric flow, Riemann surfaces, Discrete meric, Circle packing metric, Discrete curvature.

\section{Introduction}

Let $\mathfrak{M}$ be $n$-dimensional Riemannian manifold with Riemannian metric $g_{i j}$. The following general evolution equations for the metric $g_{i j}$ and Ricci curvature $R_{i j}$

$$
\frac{\partial^{2} g_{i j}}{\partial t^{2}}=-2 R_{i j}
$$

Received August 31, 2010

MSC(2010): 14H55, 37E35, 53C44,52C26.

* This work is partially supported by NUDT Preparing Research Project (JC110204) and National Natural Science Foundation of China (No. 11001237) 
have been introduced recently by Kong and Liu motivated by Einstein equation and Ricci flow [1-5] and named hyperbolic geometric flow(HGF), which can be used to describe the wave character of metrics and curvatures of manifolds and carries many interesting features of both the Ricci flow as well as the Einstein equations [6-14]. This is essentially different from the Ricci flow, which describe the heat diffusion process character of metrics and curvatures.

In this paper, we mainly focus on the numerical computation of the initial problem of HGF on closed Riemann surfaces, namely a compact manifold without boundary, such as sphere, torus and double torus. The reason for calculating on closed manifold is because that this surface has a triangulation with a finite number of triangles, and no need to consider boundary conditions, e.g. Dirichlet conditions. As the first part of our research on this topic, we are interested in the computation using Thurston's circle packing on surfaces. This is based on works of the discrete Ricci flow on piecewise linear surfaces introduced by $\mathrm{Gu}$ et al [15-18]. As the second part of our research, we are interested in the pointwise conformal class $\left(\mathfrak{M}, w(t, \cdot) g_{0}\right)$, where $w(t, \cdot)$ is a nonnegative function with parameter $t$ and $g_{0}$ is the initial metric on the surface. Therefore, the Ricci curvature is

$$
R=\frac{2}{w}\left(k-\frac{1}{2} \Delta_{g_{0}} \ln w\right)
$$

where $k$ is the Gaussian curvature of $\left(\mathfrak{M}, g_{0}\right)$. Thus, HGF can be deduced to

$$
\frac{\partial^{2} w}{\partial t^{2}}=\Delta_{g_{0}} \ln w-2 k
$$

We give a numerical algorithm for initial value problem of Eq.(2) on closed Riemann surface, such as sphere and tours. The numerical experiments verify the qualitative conclusions presented by Kong and Liu et al, including the theorem: if the Euler characteristic number of surface is positive, then any solution of Eq.(2) must decay in finite time for any initial problem. 


\section{Computation of HGF for circle packing metric}

\section{Computational domain}

A closed Riemann surface has a triangulation with a finite number of triangles, and the Riemannian metric and the Gaussian curvature are discretized as the edge lengths and the angle deficits. In this section, we compute the HGF with circle packing metric. This method is same as discrete Ricci flow, except the different for derivative of time. Consider a triangle mesh $\Sigma$ for $\mathfrak{M}$ with vertices $V$, edges $E$ and triangles $F$. If each triangle in $F$ is realizable on the Euclidean plane, then we call $\Sigma$ is with Euclidean background geometry. Similarly, the meshes can be defined with spherical or hyperbolic background geometries respectively. Some concepts in differential geometry, such as metric, Gaussian curvature, and conformal structure can also be defined on mesh.

\section{Discrete Riemannian metric}

Consider a triangle $f_{i j k}$ with edge lengths $l_{i j}, l_{j k}, l_{k i}$, and the angles $\theta_{k}, \theta_{i}, \theta_{j}$ against the corresponding edges. The edge lengths define the discrete Riemannian metric on $\Sigma$ by

$$
l: E \rightarrow \mathbb{R}^{+},
$$

such that for a triangle $f_{i j k}$ the edge lengths satisfy the triangle inequality

$$
l_{i j}+l_{j k}>l_{k i} .
$$

\section{Discrete Gaussian curvature}

The discrete Gaussian curvature $K_{i}$ on a vertex $v_{i}$ can be computed using Gaussian Bonnet formula from the angle deficit:

$$
K_{i}=2 \pi-\sum_{f_{i j k} \in F} \theta_{i}^{j k}
$$

where $\theta_{i}^{j k}$ represents the corner angle attached to vertex $v_{i}$ in the face $f_{i j k}$. The values of angles and edge lengths are related by cosine laws. Meshes with Eu- 
clidean background geometries require the cosine laws as follows:

$$
l_{i j}^{2}=l_{j k}^{2}+l_{k i}^{2}-2 l_{j k} l_{k i} \cos \theta_{k},
$$

where $l_{i j}, l_{j k}, l_{k i}$ are edges in a triangle $f_{i j k}$ and $\theta_{k}$ is the angle attached to vertex $v_{k}$.

\section{Discrete conformal structure}

Let $\Gamma$ be a function defined on the vertices

$$
\Gamma: V \rightarrow \mathbb{R}^{+}
$$

which assigns a radius $r_{i}$ to the vertex $v_{i}$. Let $\Phi$ be a function defined on the edges,

$$
\Phi: E \rightarrow\left[0, \frac{\pi}{2}\right],
$$

which assigns an acute angle $\Phi\left(l_{i j}\right)$ to each edge $l_{i j}$. The pair $(\Gamma, \Phi)$ on the mesh $\Sigma$ is called a circle packing metric of $\Sigma$. Two circle packing metrics $\left(\Gamma_{1} ; \Phi_{1}\right)$ and $\left(\Gamma_{2} ; \Phi_{2}\right)$ on a same mesh are conformal equivalent, if $\Phi_{1}=\Phi_{2}$.

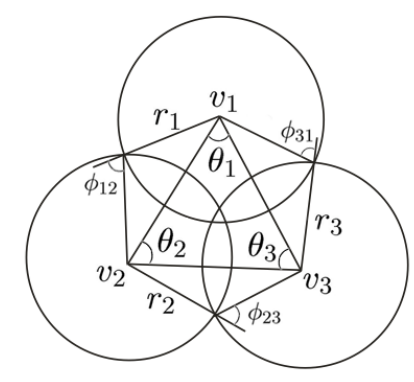

Figure 1: Circle packing metric for a triangle

The circle packing metric and the edge lengths can be converted to each other by using cosine laws dependent on the Euclidean background geometry as follows:

$$
l_{i j}^{2}=r_{i}^{2}+r_{j}^{2}+2 r_{i} r_{j} \cos \phi_{i j}
$$

where $v_{i}$ has a circle whose radius is $r_{i}, \phi_{i j}$ is defined by the two circles of $v_{i}$ and $v_{j}$ (see Fig.1). 


\section{Discrete HGF}

Now, we are ready to derive the discrete $\mathrm{HGF}$ (DHGF) on surfaces, which is an system of evolution difference equations of the discrete metric. Let

$$
u_{i}=\log r_{i}
$$

Since the discrete Ricci flow [18] is defined as:

$$
\frac{d u_{i}(t)}{d t}=-2 K_{i}
$$

we can similarly derive the DHGF as:

$$
\frac{d^{2} u_{i}(t)}{d t^{2}}=-2 K_{i}
$$

The temporal partial derivatives can be approximated by differences. The computational scheme for Eqs.(6) is

$$
u_{i}^{n+1}=-2(\Delta t)^{2} K_{i}^{n}+2 u_{i}^{n}-u_{i}^{n-1} .
$$

where $u_{i}^{n}$ denotes $u_{i}(n \Delta t)$.

\section{Geometric properties}

The first geometric property is that the metrics on surfaces at each time step is conformal to the original metrics, since $\Phi$ is invariant in the computational progresses. The other geometric property is that the Euler characteristic number has essential relationship with the solution for Eqs.(7). More accurately, we have

Theorem 2.1 Let $\chi(\mathfrak{M})$ be the Euler characteristic number for closed Riemann surface $\mathfrak{M}$ with triangulation $\Sigma$. If $\left\{u_{i}^{n} \mid i \in V\right\}$ is a solution of Eqs.(2), then

a. If $\chi(\mathfrak{M})<0$, then the discrete metric for Eqs.(7) expand in infinite time for any initial value $\left\{u_{i}^{0}, u_{i}^{1} \mid i \in V\right\}$.

b. If $\chi(\mathfrak{M})=0$ and $u_{i}^{1}<u_{i}^{0}$ for all $i \in V$, then the discrete metric for Eqs.(7) decay in infinite time; 
c. If $\chi(\mathfrak{M})>0$, then the discrete metric for Eqs.(7) decay in infinite time for any initial value $\left\{u_{i}^{0}, u_{i}^{1} \mid i \in V\right\}$.

Proof. Taking integration on both sides of Eqs.(7) and using Gauss-Bonnet formula, we have

$$
\sum_{i \in V} u_{i}^{n}=-2 n(n-1) \pi \chi(\mathfrak{M}) \Delta t^{2}+n \sum_{i \in V} u_{i}^{1}-(n-1) \sum_{i \in V} u_{i}^{0}
$$

for $n \geqq 2$ and any initial values $\left\{u_{i}^{1}, u_{i}^{0} \mid i \in V\right\}$. The conclusions (a-c) can be concluded from (5) and (8).

It can be see that Euler character is the essential geometric obstruction to the existence of periodic solution on sphere and double torus.

\section{Algorithm}

The numerical algorithm to compute Riemannian metrics with prescribed Gaussian curvatures using DHGF is designed. The unified pipeline for this algorithm is listed as follows:

\section{Step 1. Compute the discrete Gaussian curvature.}

This curvature can be computed from Eq.(2) using Gaussian Bonnet formula.

\section{Step 2. Compute the initial circle packing metric.}

2.1. For each face $f_{i j k}$, compute a radius for the vertex $v_{i}$

$$
r_{i}^{j k}=\frac{l_{k i}+l_{i j}-l_{j k}}{2},
$$

where $l_{i j}, l_{j k}$, and $l_{k i}$ are the lengths of the edges $e_{i j}, e_{j k}$, and $e_{k i}$, respectively.

2.2. For each vertex $v_{i}$, approximate the radius $r_{i}$ by averaging the radius from the faces adjacent to $v_{i}$ :

$$
r_{i}=\frac{1}{m} \sum_{f_{i j k \in F}} r_{i}^{j k},
$$

where $m$ is the number of the adjacent faces to vertex $v_{i}$. 
2.3. For each edge $l_{i j}$, compute its edge weight $\phi_{i j}$ from $r_{i}, r_{j}$ using a cosine law (4). If the edge weight is greater than $\frac{\pi}{2}$, take $\frac{\pi}{2}$ as its value.

\section{Step 3. Update the circle pacing metric by DHGF.}

3.1. Compute edge lengths $l_{i j}$ from the current vertices radius $r_{i}$ and $r_{j}$ and the fixed edge weight $\phi_{i j}$ using the cosine law (4).

3.2. Compute the corner angles $\theta_{i}$ in each face $f_{i j k}$ from the current edge lengths by using the cosine law (3).

3.3. Compute the discrete Gaussian curvature $K_{i}$ of each vertex $v_{i}$ by using Eq.(2).

3.4. Update $u_{i}$ of each vertex $v_{i}$ by Eqs.(7).

3.5. Update $r_{i}$ by Eqs.(5), and go to Step 3.1.

The following numerical experiments in this section show that this algorithm is not a very efficient algorithm for practical use due to the lowly convergence.

\section{Examples}

This numerical algorithm has been implemented in Java on a PC with AMD CPU of $3.4 \mathrm{GHz}$ and 2GB RAM. We use Hypermesh to generat the triangle meshes for sphere, torus, and double torus(Fig.2).
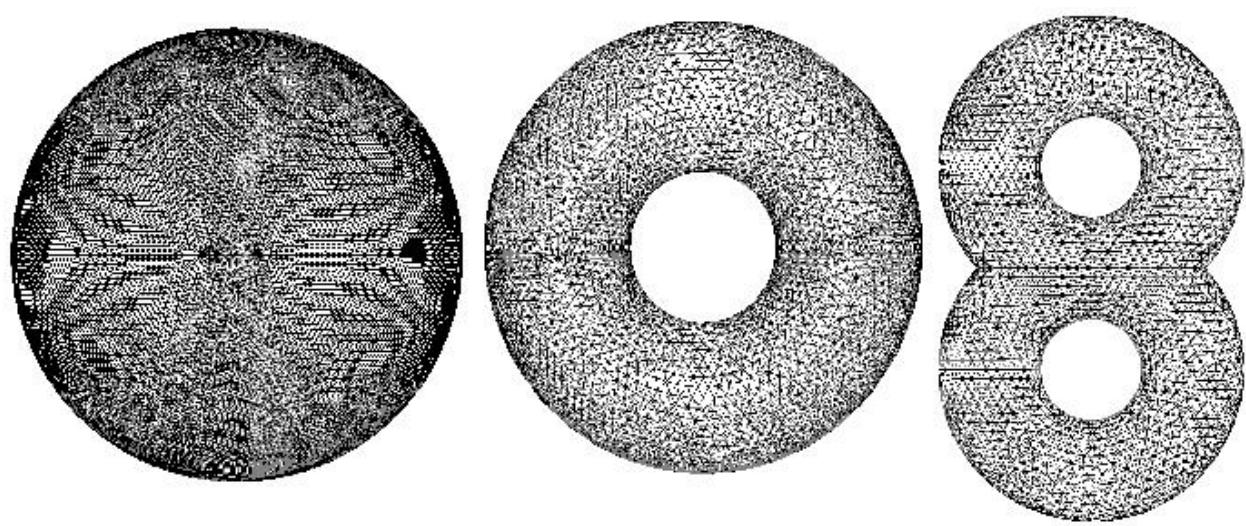

Figure 2: Triangle meshes for sphere, torus and double torus 
For those meshes, we can calculate the initial value for $\left\{u_{i}^{0}, i \in V\right\}$ and let $u_{i}^{0}=u_{i}^{1}$, for all $i \in V$, namely let the initial velocity be zero. Then we obtain a initial value problem for Eqs.(7) on sphere, torus and double torus.

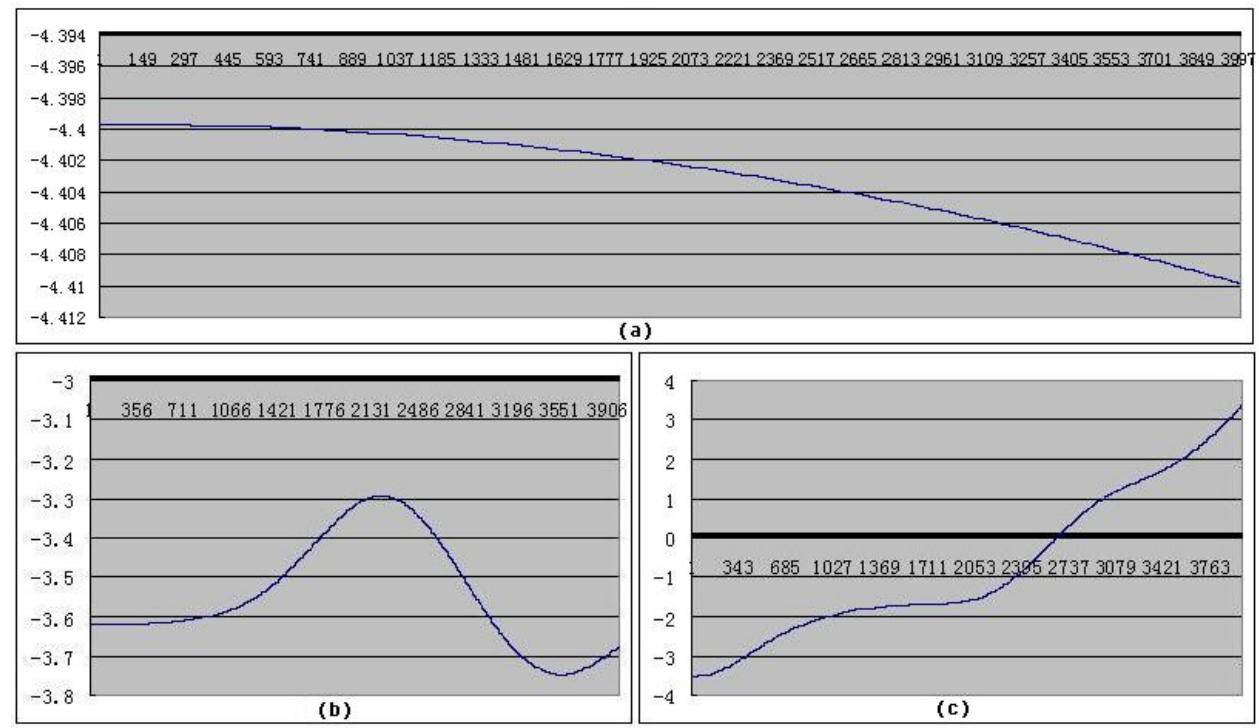

Figure 3: The trajectory of $u_{i}$ under DHGF on different surfaces

Fig.3(a) shows the trajectory of one $u_{i}$ in $\left\{u_{i} \mid i \in V\right\}$ on sphere for this initial value problems, (b) for torus, and (c) for double torus. Since the value of $u_{i}$ on a vertex $i$ can not give the information of others in $\left\{u_{i} \mid i \in V\right\}$, we let $u$ be the mean value of $\left\{u_{i} \mid i \in V\right\}$ to describe the behavior of all $u_{i}$ and record the initial and end values of $u$. Table 1 recodes the data of initial and end values of $u$ for a initial value problem on sphere.

\begin{tabular}{|c|c|c|c|c|}
\hline$\Delta t$ & $\mathrm{~N}$ & $u^{0}=u^{1}$ & $u^{N}$ & $r^{N}$ \\
\hline 0.0245 & 877 & -4.399645598 & -5.586009882 & 0.003778194 \\
\hline 0.001 & 3999 & -4.399645598 & -4.409869389 & 0.012251197 \\
\hline 0.0001 & 3999 & -4.399645598 & -4.399747835 & 0.012376393 \\
\hline 0.00001 & 3999 & -4.399645598 & -4.3996466198 & 0.0123776606 \\
\hline
\end{tabular}

Table 1: Data for a initial value problem on sphere

The data in Table 1 and conclusion in Theorem 2.1(c) show that the discrete metric tend to 0 in infinite time. Since the Euler characteristic number of sphere 
is 2 and the initial value satisfies $u^{1}=u^{0}<0$, the trajectory of $u$ is a parabola by Theorem 2.1(c). In Fig.4, it can be seen that the trend line for trajectory of $u$ likes a parabola $y=a x^{2}+b, a<0, b<0$. Fig.4(a) is the trajectory of $u$ with $\Delta t=0.024527068$, (b) with $\Delta t=0.001$, (c) with $\Delta t=0.0001$ and (d) with $\Delta t=0.00001$.

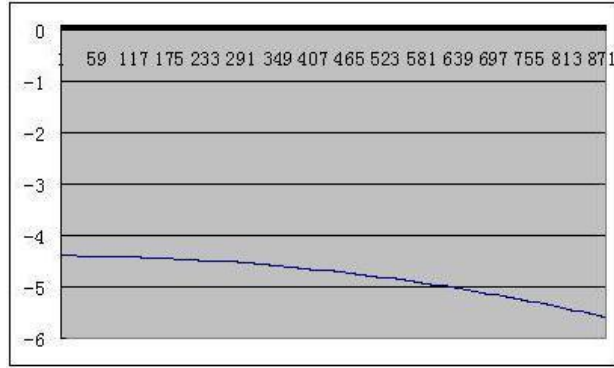

(a)

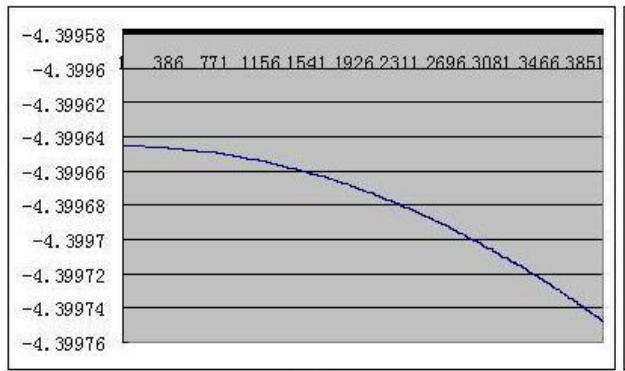

(c)

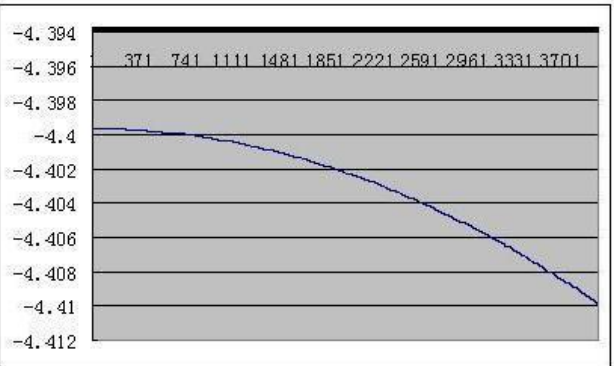

(b)

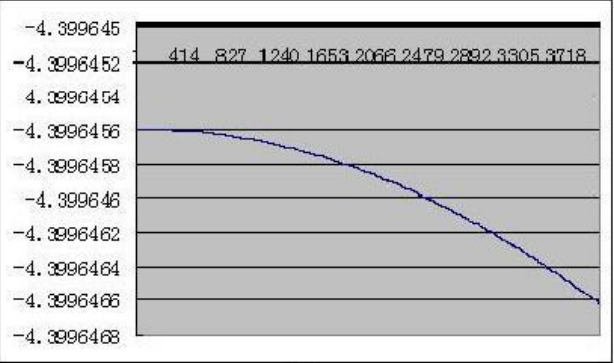

(d)

Figure 4: The trajectory of $u$ under DHGF on sphere

Table 2 recodes the data of initial and end values of $u$ for a initial value problem on double torus.

\begin{tabular}{|c|c|c|c|c|}
\hline$\Delta t$ & $\mathrm{~N}$ & $u^{0}=u^{1}$ & $u^{N}$ & $r^{N}$ \\
\hline 0.01 & 3999 & -3.074213079 & 3.404914496 & 32.27251434 \\
\hline 0.001 & 3999 & -3.074215499 & -3.009424208 & 0.051216977 \\
\hline 0.003 & 3999 & -3.074215435 & -2.491093808 & 0.124428483 \\
\hline 0.0005 & 3999 & -3.074215506 & -3.058017683 & 0.047438238 \\
\hline
\end{tabular}

Table 2: Data for a initial value problem on double torus

Since the Euler characteristic number of double torus is -2 and the initial value satisfies $u^{1}=u^{0}<0$, the trajectory of $u$ is a parabola like $y=a x^{2}+b, a>$ 
$0, b<0$ by Theorem 2.1(a). In Fig.5, it can be seen that the trend line for trajectory of $u$ is a parabola. Fig.5(a) is the trajectory of $u$ with $\Delta t=0.01$, (b) with $\Delta t=0.001$, (c) with $\Delta t=0.003$ and (d) with $\Delta t=0.0005$.
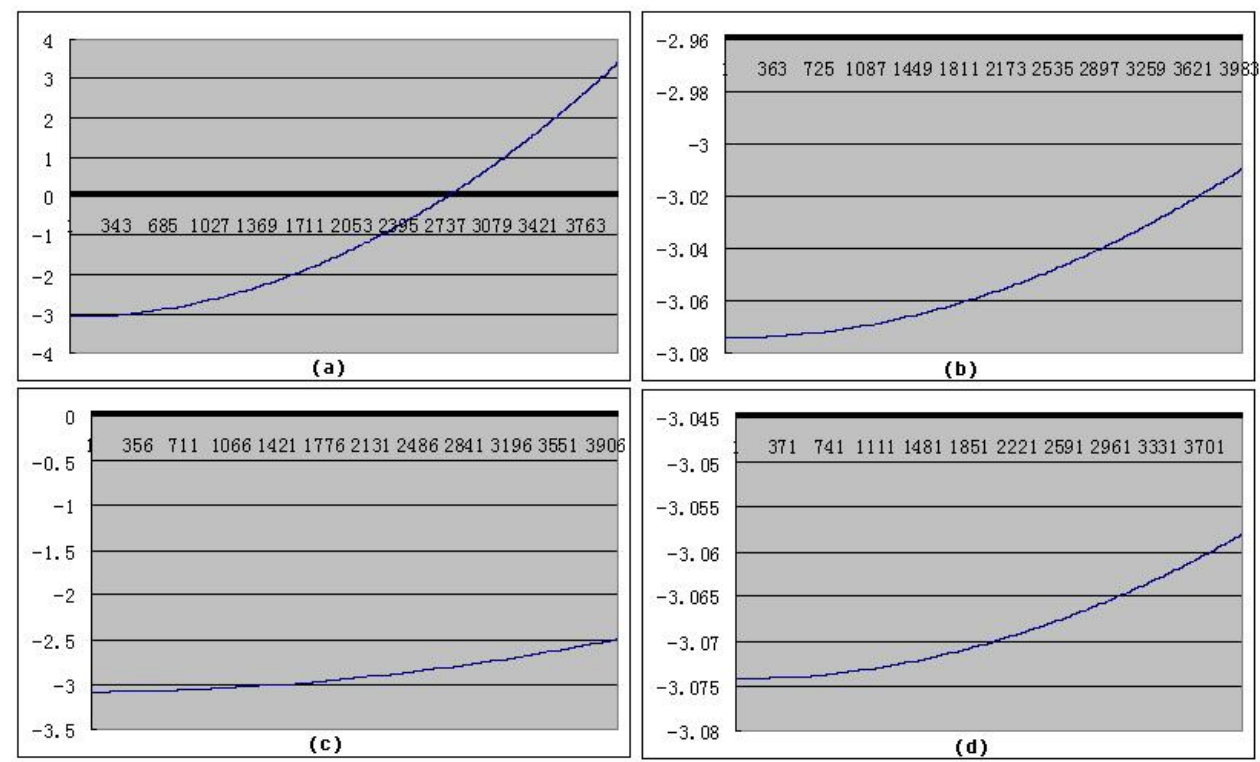

Figure 5: The trajectory of $u$ under DHGF on double torus

Table 3 recodes the data of initial and end values of $u$ for a initial value problem on torus.

\begin{tabular}{|c|c|c|c|c|}
\hline$\Delta t$ & $\mathrm{~N}$ & $u^{0}=u^{1}$ & $u^{N}$ & $r^{N}$ \\
\hline 0.001 & 3999 & -3.692617641 & -3.692617641 & 0.025714392 \\
\hline 0.0001 & 3999 & -3.692617641 & -3.692617641 & 0.025714392 \\
\hline 0.0005 & 3999 & -3.692617641 & -3.692617641 & 0.025714392 \\
\hline 0.00001 & 3999 & -3.692617641 & -3.692617641 & 0.025694951 \\
\hline
\end{tabular}

Table 3: Data for a initial value problem on torus

Since the Euler characteristic number of torus is 0 and the initial value satisfies $u^{1}=u^{0}<0$, the trajectory of $u$ is a straight line like $y=b, b<0$ by Theorem 2.1(b). In Fig.6, it can be seen that the trend line for trajectory of $u$ is a straight line. Fig.6(a) is the trajectory of $u$ with $\Delta t=0.001$, (b) with $\Delta t=0.0001$, (c) 
with $\Delta t=0.0005$ and (d) with $\Delta t=0.00001$.

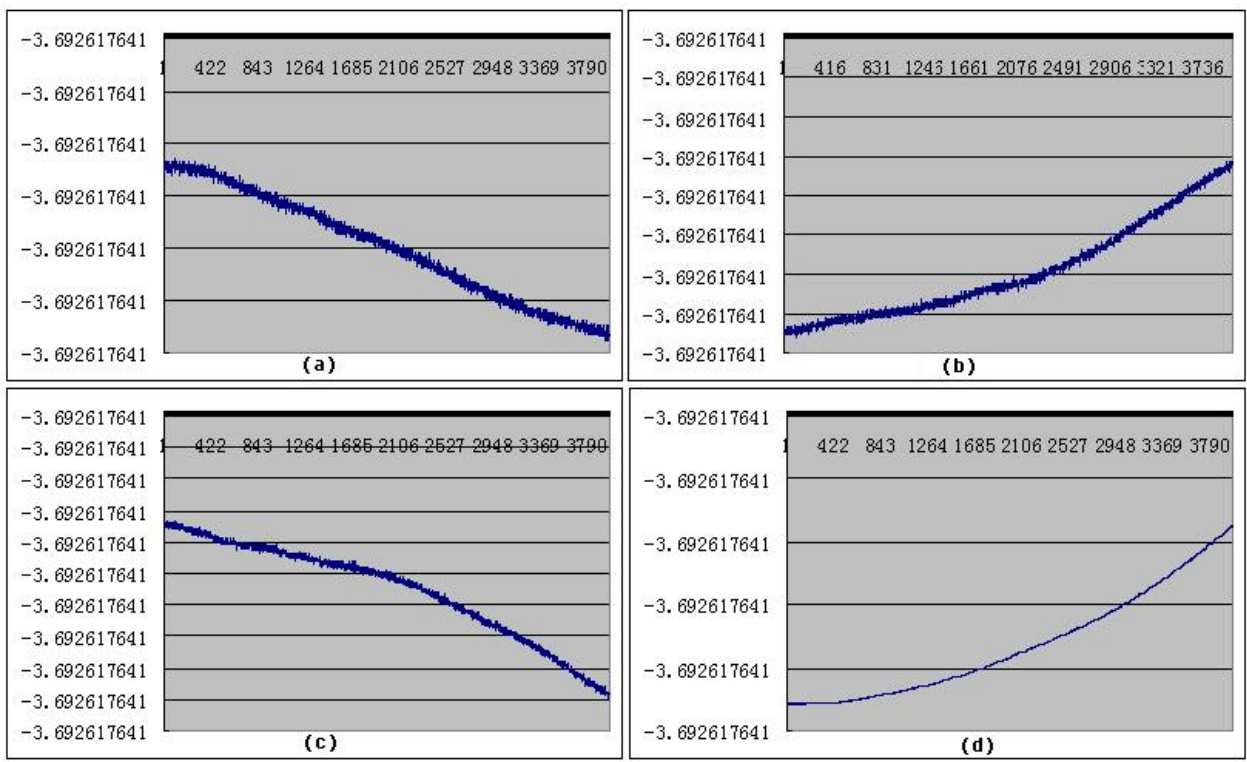

Figure 6: The trajectory for $u$ of DHGF on torus

Based on data in Tables (1-3), we can obtain the qualitative behavior of mean value of discrete metric for three initial problems of DHGF (7) on different surfaces in Table 4.

\begin{tabular}{|c|c|c|c|c|}
\hline $\begin{array}{c}\text { Riemann } \\
\text { sufaces }\end{array}$ & $\begin{array}{c}\text { Euler } \\
\text { characteristic }\end{array}$ & $\begin{array}{c}\text { Initial discrete } \\
\text { metric }\end{array}$ & $\begin{array}{c}\text { Initial } \\
\text { velocity }\end{array}$ & $\begin{array}{c}\text { Mean value } \\
\text { of metric }\end{array}$ \\
\hline Sphere & 2 & $<1$ & 0 & Decay \\
\hline Torus & 0 & $<1$ & 0 & Invariant \\
\hline Double torus & -2 & $<1$ & 0 & Expand \\
\hline
\end{tabular}

Table 4 : Summary of numerical results 


\section{Computation of HGF for pointwise conformal met- ric}

\section{Computational scheme}

As the second part of our research, we are interested in calculating HGF on Riemann surfaces with pointwise conformal metric, namely the simplified HGF (2). The 2D space manifold can be approximated by triangles, and the time by line segments. We suppose each simplex contains its circumcenter and establish a discrete scheme for Eqs.(2) on triangles in Fig.7 as an example for a mesh, in which $0, \ldots, C$ are vertices, $1,2,3$ are the circumcenters of triangles, $a, b, c$ are the circumcenters of edges. Letting $l_{i j}$ be the length of line segment $(i, j)$ and $A_{i j k l}$ be the area of quadrangle $(i, j, k, l)$, we approximate Eqs.(2) as follows:

$$
\begin{aligned}
w_{O}^{n+1}= & 2 w_{O}^{n}-w_{O}^{n-1}-\frac{2 \Delta t^{2}}{P_{O}}(2 \pi-\angle A O B-\angle A O C-\angle B O C)+\frac{\Delta t^{2}}{P_{O}}\left(\frac{l_{13}}{l_{A O}}\right. \\
& \left.\times\left(\ln w_{A}^{n}-\ln w_{O}^{n}\right)+\frac{l_{12}}{l_{B O}}\left(\ln w_{B}^{n}-\ln w_{O}^{n}\right)+\frac{l_{23}}{l_{C O}}\left(\ln w_{C}^{n}-\ln w_{O}^{n}\right)\right) .
\end{aligned}
$$

where $l_{12}:=l_{1 b}+l_{2 b}, l_{23}:=l_{2 c}+l_{3 c}, l_{31}:=l_{3 a}+l_{1 a}$ and $P_{O}:=A_{O 1 a b}+A_{O 2 b c}+$ $A_{O 3 a c}$.

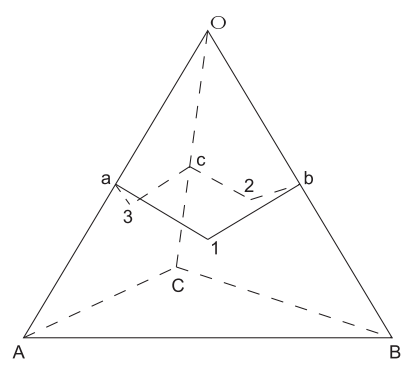

Figure 7: A part of triangle mesh 


\section{Analysis of stability}

Suppose there is a perturbation $\varepsilon_{i}^{n}$ on $w_{i}^{n-1}$ and $w_{i}^{n}$ on each vertice $i$, the relation between $\varepsilon_{i}^{n}$ and $\varepsilon_{i}^{n+1}$ can be induced from Eqs.(9) as follows:

$$
\begin{aligned}
\varepsilon_{O}^{n+1} \approx & \varepsilon_{O}^{n}+\frac{\Delta t^{2}}{P_{O}}\left(\frac{l_{13}}{l_{A O}}\left(\frac{\varepsilon_{A}^{n}}{w_{A}^{n}}-\frac{\varepsilon_{O}^{n}}{w_{O}^{n}}\right)+\frac{l_{12}}{l_{B O}}\left(\frac{\varepsilon_{B}^{n}}{w_{B}^{n}}-\frac{\varepsilon_{O}^{n}}{w_{O}^{n}}\right)+\frac{l_{23}}{l_{C O}}\left(\frac{\varepsilon_{C}^{n}}{w_{C}^{n}}-\frac{\varepsilon_{O}^{n}}{w_{O}^{n}}\right)\right) \\
< & \varepsilon_{O}^{n}-\frac{\Delta t^{2}}{P_{O}}\left(\frac{l_{13}}{l_{A O}}+\frac{l_{12}}{l_{B O}}+\frac{l_{23}}{l_{C O}}\right)\left(\frac{\varepsilon_{O}^{n}}{\max w_{i}^{n}}-\frac{\max \varepsilon_{i}^{n}}{\min w_{i}^{n}}\right) \\
= & \left(1-\frac{\Delta t^{2}}{P_{O}}\left(\frac{l_{13}}{l_{A O}}+\frac{l_{12}}{l_{B O}}+\frac{l_{23}}{l_{C O}}\right) \frac{1}{\max w_{i}^{n}}\right) \varepsilon_{O}^{n} \\
& +\frac{\Delta t^{2}}{P_{O}}\left(\frac{l_{13}}{l_{A O}}+\frac{l_{12}}{l_{B O}}+\frac{l_{23}}{l_{C O}}\right) \frac{\max \varepsilon_{i}^{n}}{\min w_{i}^{n}} .
\end{aligned}
$$

Therefore, we can say Eqs.(9) is unstable because

$$
1-\frac{\Delta t^{2}}{P_{O}}\left(\frac{l_{13}}{l_{A O}}+\frac{l_{12}}{l_{B O}}+\frac{l_{23}}{l_{C O}}\right)\left(\frac{1}{\max w_{i}^{n}}-\frac{1}{\min w_{i}^{n}}\right)>1 .
$$

So we should choose very small time step to avoid numerical divergence rapidly. If the initial metric, initial velocity, and Gaussian curvature are isotropic, Eqs.(9) in this case is almost stable since $\max w_{i}^{n} \approx \min w_{i}^{n}$. The numerical experiment (Fig.8, Table.5) also verifies this point.

\section{Geometric properties}

The geometric property of Eqs.(9) shows that the Euler characteristic number has essential relationship with the solution. More specifically, we have

Theorem 3.1 Let $\chi(\mathfrak{M})$ be the Euler characteristic number for closed Riemann surface $\mathfrak{M}$ with triangulation $\Sigma$. If $\left\{w_{i}^{n} \mid i \in V\right\}$ is a solution of Eqs.(9), then

a. If $\chi(\mathfrak{M})>0$, then any solution of Eqs.(9) decay in finite time for any initial value $\left\{w_{i}^{0}, w_{i}^{1} \mid i \in V\right\}$.

b. If $\chi(\mathfrak{M})=0$ and $w_{i}^{1}<w_{i}^{0}$ for all $i \in V$, then solutions of Eqs.(9) decay in finite time;

c. If $\chi(\mathfrak{M})<0$ and $w_{i}^{1} \geq w_{i}^{0}$ for all $i \in V$, then solutions of Eqs.(9) expand in infinite time. 
Proof. Taking integration on both sides of Eqs.(9) and using Gauss-Bonnet formula, we have

$$
\sum_{i \in V} w_{i}^{n} P_{i}=-2 n(n-1) \pi \chi(\mathfrak{M}) \Delta t^{2}+n \sum_{i \in V} w_{i}^{1} P_{i}-(n-1) \sum_{i \in V} w_{i}^{0} P_{i}
$$

for $n \geqq 2$ and any initial conditions $\left\{w_{i}^{1}, w_{i}^{0} \mid i \in V\right\}$. The conclusions (a-c) can be concluded from the expression (10).

From the Theorem 3.1, we see that Eqs.(9) have essential geometric obstruction to the existence of periodic solution on sphere and double torus.

\section{Examples}

Table 5 records the value of one $w_{i}$ in $\left\{w_{i} \mid i \in V\right\}$ on sphere for a special initial problems of Eqs. (9) with time step $\Delta t=6.934 \times 10^{-6}$. Since the value of $w_{i}$ can not give the information of others in $\left\{w_{i} \mid i \in V\right\}$, we let $w$ be the mean value of $\left\{w_{i} \mid i \in V\right\}$ to describe the behavior of all $w_{i}$ and record the value of $w$.

\begin{tabular}{|c|c|c|c|c|c|}
\hline$N$ & $w_{i-1}^{N}$ & $w_{i}^{N}$ & $\Delta \ln w_{i}^{N}$ & $k_{i}^{N}$ & $w^{N}$ \\
\hline 1 & 1 & 1 & 0 & $1.53 \times 10^{-7}$ & 1 \\
\hline 952 & 0.928424 & 0.928575 & $-1.23 \times 10^{-8}$ & $1.53 \times 10^{-7}$ & 0.931719 \\
\hline 1903 & 0.708709 & 0.709017 & $1.60 \times 10^{-9}$ & $1.53 \times 10^{-7}$ & 0.72869 \\
\hline 2854 & 0.356898 & 0.357318 & $7.72 \times 10^{-8}$ & $1.53 \times 10^{-7}$ & 0.392946 \\
\hline 3488 & 0.0741055 & 0.0745791 & $1.07 \times 10^{-8}$ & $1.53 \times 10^{-7}$ & 0.0972911 \\
\hline
\end{tabular}

Table 5: Data for a initial value problem of Eqs.(9) on sphere

Since the Euler characteristic number of sphere is 2 and the initial value satisfies $w^{1}=w^{0}>0$, the trajectory of $\sum_{i \in V} w_{i}^{n} P_{i}$ is a parabola like $y=a x^{2}+b, a<$ $0, b>0$ by Theorem 3.1(a). So $w$ is almost a parabola if $P_{i} \approx P_{j}, \forall i, j \in V$. Fig.8(a) is the trajectory of one $w_{i}$ and (b) is a trajectory of $w$. It can be seen 
that the trend line in Fig.8(b) is a parabola.
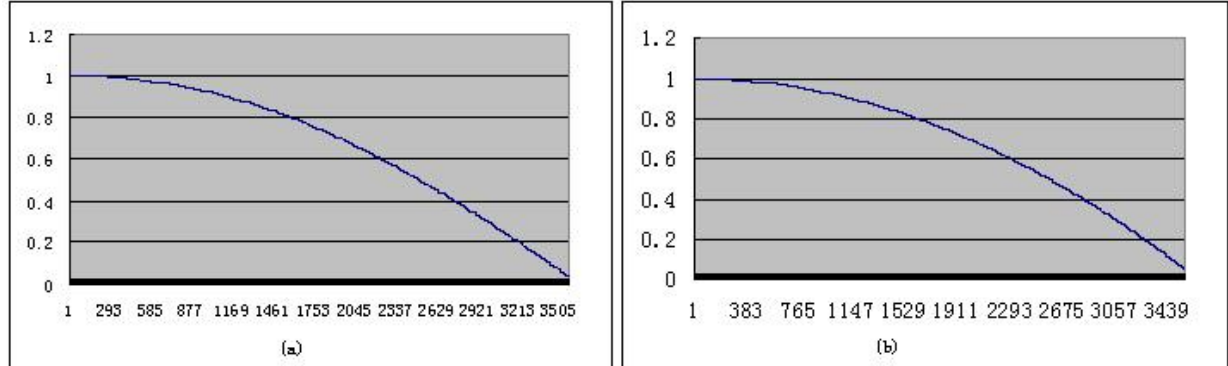

Figure 8: The trajectory of a $w_{i}$ and $w$ of Eqs.(9) on sphere

Table 6 shows the data for a special initial problems for Eqs.(9) on torus with time step $\Delta t=2.8311 \times 10^{-5}$.

\begin{tabular}{|c|c|c|c|c|c|}
\hline$N$ & $w_{i-1}^{N}$ & $w_{i}^{N}$ & $\Delta \ln w_{i}^{N}$ & $k_{i}^{N}$ & $w^{N}$ \\
\hline 1 & 1 & 1 & 0 & $7.83 \times 10^{-8}$ & 1 \\
\hline 1255 & 0.929713 & 0.929832 & $-1.58 \times 10^{-8}$ & $7.83 \times 10^{-8}$ & 0.957312 \\
\hline 2509 & 0.437896 & 0.438117 & $7.53 \times 10^{-7}$ & $7.83 \times 10^{-8}$ & 1.00795 \\
\hline 3345 & 0.432457 & 0.432378 & $7.95 \times 10^{-7}$ & $7.83 \times 10^{-8}$ & 1.02469 \\
\hline 4366 & 0.438288 & 0.438177 & $1.02 \times 10^{-6}$ & $7.83 \times 10^{-8}$ & 0.910947 \\
\hline
\end{tabular}

Table 6 : Data for a initial value problem of Eqs.(9) on torus

Since the Euler characteristic number of torus is 0 and the initial value satisfies $w^{1}=w^{0}>0$, the trajectory of $\sum_{i \in V} w_{i}^{n} P_{i}$ is a line like $y=b, b>0$ by Theorem 3.1(b). So $w$ is almost a line if $P_{i} \approx P_{j}, \forall i, j \in V$. Fig.9(a) is the trajectory of one $w_{i}$ in $\left\{w_{i} \mid i \in V\right\}$ and (b) is a trajectory of $w$. It can be seen that the trend line for trajectory of $w$ is almost a line. The trajectory in Fig.9 (b) is not exact a line, in part because the anisotropy of the curvature and the irregularity of mesh. The value of $w$ will neither decay nor expand. If $w_{i}^{+\infty}=+\infty$, then $\sum_{i \in V} w_{i}^{+\infty} P_{i}=+\infty$, which contradicts to Theorem 3.1(b). If there is a $N$ such that $w_{i}^{N}=0$ and $w_{j}^{N} \neq 0$ for a pair of adjacent points $i, j$, then $\Delta \ln w_{j}^{N}=\infty$, so 
$w_{j}^{N+1}=\infty$, which contradicts with Theorem 3.1(b). So $w$ will always oscillate.
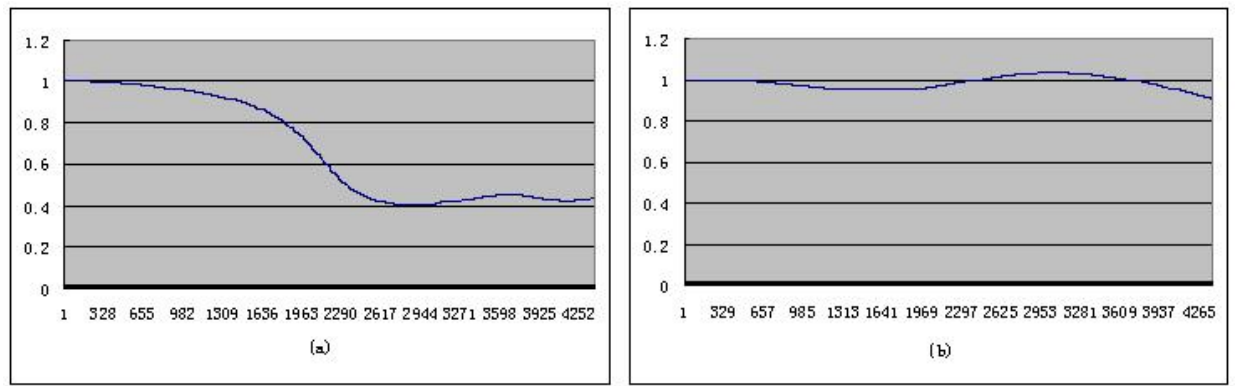

Figure 9: The trajectory of a $w_{i}$ and $w$ of Eqs.(9) on torus

Table 7 records the data for a special initial problems for Eqs.(9) on double torus with time step $\Delta t=2.8311 \times 10^{-5}$.

\begin{tabular}{|c|c|c|c|c|c|}
\hline$N$ & $w_{i-1}^{N}$ & $w_{i}^{N}$ & $\Delta \ln w_{i}^{N}$ & $k_{i}^{N}$ & $w^{N}$ \\
\hline 1 & 1 & 1 & 0 & $1.48 \times 10^{-8}$ & 1 \\
\hline 4000 & 0.922261 & 0.922296 & $3.48 \times 10^{-9}$ & $1.48 \times 10^{-8}$ & 1.03876 \\
\hline 8000 & 0.724934 & 0.724994 & $8.16 \times 10^{-9}$ & $1.48 \times 10^{-8}$ & 1.15113 \\
\hline 12000 & 0.487802 & 0.48785 & $3.75 \times 10^{-8}$ & $1.48 \times 10^{-8}$ & 1.34347 \\
\hline 14629 & 0.451264 & 0.45125 & $3.23 \times 10^{-8}$ & $1.48 \times 10^{-8}$ & 1.51792 \\
\hline
\end{tabular}

Table 7 : Data for a initial value problem of Eqs.(9) on torus

Since the Euler characteristic number of double torus is -2 and the initial value satisfies $w^{1}=w^{0}>0$, the trajectory of $\sum_{i \in V} w_{i}^{n} P_{i}$ is a parabola like $y=a x^{2}+$ $b, a>0, b>0$ by Theorem 3.1(c). So $w$ is almost a parabola if $P_{i} \approx P_{j}$, $\forall i, j \in V$. Fig.10(a) is the trajectory of one $w_{i}$ and (b) is a trajectory of $w$. It 
can be seen that the trend line for trajectory of $w$ is almost a parabola.
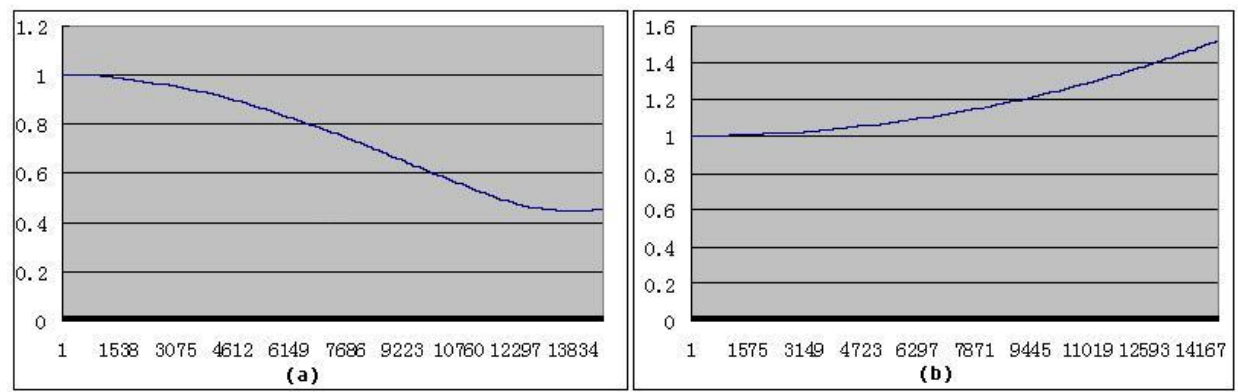

Figure 10: The trajectory of a $w_{i}$ and $w$ of Eqs.(9) on double torus

Based on data in Tables (5-7), we can obtain the qualitative behavior of mean value of discrete metric for three initial problems for Eqs.(9) on different surfaces in Table 8. It can be see that our numerical results verify the conclusion of Kong and Liu et al: if the Euler characteristic number of surface is positive, then any solution of Eq.(2) must blow up in finite time for any initial problem [11].

\begin{tabular}{|c|c|c|c|c|}
\hline $\begin{array}{c}\text { Riemann } \\
\text { sufaces }\end{array}$ & $\begin{array}{c}\text { Euler } \\
\text { characteristic }\end{array}$ & $\begin{array}{c}\text { Initial discrete } \\
\text { metric }\end{array}$ & $\begin{array}{c}\text { Initial } \\
\text { velocity }\end{array}$ & $\begin{array}{c}\text { Mean value } \\
\text { of metric }\end{array}$ \\
\hline Sphere & 2 & $>0$ & 0 & Decay \\
\hline Torus & 0 & $>0$ & 0 & Oscillation \\
\hline Double torus & -2 & $>0$ & 0 & Expand \\
\hline
\end{tabular}

Table 8: Summary of numerical results

\section{Conclusion}

We have introduced two algorithms for HGF with circle packing metric and pointwise conformal metric respectively that allow to study HGF in computing view. The experimental results are consistent with the theoretical achievement by Kong and Liu. This proofs the correctness and effectiveness of those algorithms at a certain extent. One limitation of the method for the experiments that we performed was the dependence of the high qualitative triangulation for Riemann surfaces, namely, each triangle should be acute one. The other limitation is the computation time. It is interesting to note that the speed of our algorithms 
can be substantially improved by using some method, for instance by Newtonian iteration.

\section{Future work}

\section{Boundary conditions}

The discrete Gaussian curvature can also be defined as the angle deficit on a boundary of meshes as

$$
K_{i}=\pi-\sum_{f_{i j k} \in F} \theta_{i}^{j k}
$$

where $\theta_{i}^{j k}$ represents the corner angle attached to vertex $v_{i}$ in the face $f_{i j k}$. Hence, we can consider the mixed initial boundary value problem for hyperbolic geometric flow.

\section{Open regions}

A consideration with numerical approach to solving HGF is that many geometries of interest are defined in open regions where the spatial domain of the computed field is unbounded in one or more coordinate directions, e.g., $\mathbb{R}^{2}$. Principally, this is because those scheme in this paper cannot be implemented at the outermost vertices, since by definition there is no information concerning the curvature value outside of computational domain.

\section{Singularities}

It well known that one can understand the heat kernel from the kernel of wave equation. This indicates that one can derive various information of the Ricci flow from that of the HGF. Therefore it is also interesting to understand the relations between the HGF and the Ricci flow, the singularities of its solutions and its relation by numerical method. This will be another interesting topic to exploration. 
Acknowledgements The author Z. Xie would like to express his deep gratitude to Professors K.F. Liu and D.X. Kong in Zhejiang University for several constructive suggestions and the useful information concerning the content of this paper.

\section{References}

[1] R.S. Hamilton, The Ricci flow on surfaces, Mathematics and general relativity, vol. 71, 237-262, (1988).

[2] R.S. Hamilton, Three-manifolds with positive Ricci curvature, J. Differential Geom. 17, 255-306, (1982).

[3] V. Fock, The theory of space, time and gravitation, second revised edition. Translated from the Russian by N. Kemmer. A Pergamon Press Book The Macmillan Co., New York, 1964.

[4] R. Penrose, Gravitational collapse and space-time singularities, Phys. Rev. Lett. 14, 57-59, (1965).

[5] H.D. Cao, X.P. Zhu, A complete proof of the Poincaré and geometrization conjectures application of the Hamilton-Perelman theory of the Ricci flow, Asian J. Math. 10, 165-492, (2006).

[6] W.-R. Dai, D.-X. Kong \& K.-F. Liu, Dissipative hyperbolic geometric flow, Asian J. Math. 12 (2008), 345-364.

[7] D.-X. Kong, Hyperbolic geometric flow, the Proceedings of ICCM 2007, Vol. II, Higher Educationial Press, Beijing, 2007, 95-110.

[8] D.-X. Kong \& K.-F. Liu, Wave character of metrics and hyperbolic geometric flow, J. Math. Phys. 48 (2007), 103508.

[9] D.-X. Kong, K.-F. Liu \& Y.-Z. Wang, Life-span of classical solutions to hyperbolic geometric flow in two space variables with slow decay initial data, to appear in Communications in Partial Differential Equations. 
[10] D.-X. Kong, K.-F. Liu \& D.-L. Xu, The hyperbolic geometric flow on Riemann surfaces, Communications in Partial Differential Equations 34 (2009), $553-580$.

[11] K.D. Xing; K.F. Liu, Wave character of metrics and hyperbolic geometric flow, Journal of Mathematical Physics, Volume 48, Issue 10, (2007).

[12] W.-R. Dai, D.-X. Kong \& K.-F. Liu, Hyperbolic geometric flow (I): shorttime existence and nonlinear stability, Pure and Applied Mathematics Quarterly (Special Issue: In honor of Michael Atiyah and Isadore Singer) 6 (2010), 331-359.

[13] A.E. Fischer and J.E. Marsden, The Einstein evolution equations as a first order quasi linear symmetric system hyperbolic system I, Commun. Math. Phys. 28, 1-38, (1972).

[14] Fu Wen Shu and You Gen Shen, Geometric flows and black holes, arXiv: gr-qc/0610030.

[15] B. Chow, F. Luo, Combinatorial Ricci flows on surfaces, J.differential geometry, 63, , 97-129, (2003).

[16] W.P. Thurston, Geometry and Topology of Three-Manifolds. Princeton lecture notes, (1976).

[17] F. Luo, Variational Principles on Triangulated Surfaces. http://arxiv.org/abs/0803.4232v1.

[18] M. Jin, J. Kim, F. Luo, X.F. Gu, Discrete surface Ricci flow, IEEE Transactions on Visualization and Computer Graphics, Volume 14, Issue 5, (2008).

[19] W.-R. Dai, D.-X. Kong \& K.-F. Liu, Hyperbolic geometric flow (I): shorttime existence and nonlinear stability, Pure and Applied Mathematics Quarterly (Special Issue: In honor of Michael Atiyah and Isadore Singer) 6 (2010), 331-359.

Zheng Xie

Department of Systems Engineering and Mathematics, 
National University of Defense Technology,

Changsha, (410073), China

E-mail: lenozhengxie@yahoo.com.cn

Zheng Ye

College of Computer Science \& Information Engineering,

Zhejiang Gongshang University,

Hangzhou, (310018), China 